\title{
The system of monitoring of epilepsy in a sleep
}

\author{
Alexey Gorbunov ${ }^{1 *}$, Vladimir Podolskiy ${ }^{1}$, and Vladimir Egorov ${ }^{1}$ \\ ${ }^{1}$ Tambov state technical university, 392000, 106, Sovetskaya str,, Tambov, Russia
}

\begin{abstract}
The slow development of the pathological process of neurodegeneration in the brain structures through appointment of appropriate medications can significantly reduce the risks of disability and improve the patient's quality of life. Currently, it is in many cases possible to carry out therapeutic treatment. For best results, the early diagnosis and instrumental monitoring of the treatment strategy, that is, the monitoring system. System analysis of information about motor activity of the patient during his sleep is likely to monitor epilepsy, including remote, and to predict the patient's condition.
\end{abstract}

\section{Relevance}

Brain disease - stroke and dementia, schizophrenia and depression, epilepsy and diseases movements and many others - are not only global economic burden to society, but also to a substantial decline in the quality of life of millions of people worldwide. Improvement of diagnostics, treatment and rehabilitation of diseases of the brain this is a problem of high priority in the medical world and its solution requires joint efforts of specialists not only in medicine, but also many others, including in engineering and IT.

Epilepsy is a chronic noncommunicable disorder of the brain that affects people of all ages. Globally, about 50 million people have epilepsy, one of the most common neurological diseases on a global scale. Most of the people suffering from this disease does not receive treatment they need, while at present, the epilepsy responds to treatment about $70 \%$ of cases. Almost $80 \%$ people with epilepsy live in low and middle income. Treatment of epilepsy in most cases is based on a daily intake of inexpensive drugs cost about $\$ 5-10$ per year. After 2 to 5 years of successful treatment, the medication can be discontinued in approximately $70 \%$ of children and $60 \%$ adults with no subsequent relapses. In addition, for successful treatment the most important is the ability to set the correct diagnosis at the earliest stages of the disease, as well as the correct therapeutic approach, because timely diagnosis has a significant influence on the development of the pathological process of neurodegeneration of brain structures through the appointment of appropriate drugs, it is significantly slowing down [1].

\section{Methods of diagnosis of epilepsy}

\subsection{Electroencephalography (EEG) and Video Electroencephalography (VEEG)}

Currently, the most common and most available instrumental method of diagnostics of brain disorders, including epilepsy is electroencephalography (EEG), which is held in the majority of cases only on an outpatient basis in all cases, only medical personnel with appropriate qualifications. This is due to the necessity of special training of the patient for the procedure, the EEG and the specifics of carrying out the procedure during which held the test sample and which can last several hours. According to the who a shortage of health personnel with relevant expertise is one of the main reasons hindering the successful fight against this disease [1]. In addition, prejudices, entrenched behind this disorder because it causes social problems that can hinder the circulation of people for treatment and the identification they have this disorder. In the end, this leads to the fact that up to three quarters of people with epilepsy may not receive the treatment they need, which can significantly improve their quality of life. Given the above, we can conclude that for a successful anti-epilepsy essential monitoring of this disease accessible to all social groups with the aim of early diagnostics and for observation of dynamics of treatment, with the aim not only of choosing the right tactics of treatment, but also minimize the side effects of antiepileptic drugs due to their prolonged use.

It is acknowledged that currently the most reliable method of instrumental diagnosis of epilepsy is the socalled night VEEG monitoring. This method is carried out during sleep of the patient and also the procedure provides for synchronized EEG with her the video recording of motor activity of the patient. The main disadvantages of this method does not allow its massive use is its high cost (at least $\$ 400$ per session) and use only in a clinical setting. In addition, typically the session the night VEEG is conducted during one night in hospital and it can make a significant interference in the measurement result is known "first night effect" in which the neural network of the left hemisphere of the brain remains in a state of increased activity, as evidenced by the researchers of Brown University [2].

\footnotetext{
*orresponding author: alexey.gorbunov@mail.ru
} 
Thus, from the point of view of Metrology, the process of instrumental diagnosis of epilepsy is reduced to the analysis of nonstationary process with a single implementation. But the acceptable accuracy of the analysis of nonstationary process can be achieved only under certain conditions, the chief of which, as shown in the work Julius S.Bendat and Allan G.Piersol [3], is the presence of an increasing ensemble of realizations of the process, i.e. as many as possible of the research conducted under identical conditions. Therefore, to improve the reliability of the study, it is necessary to spend some times under the same conditions, which is currently impossible.

\subsection{Studies based on the analysis of motor activity during sleep}

Research conducted at the University of Minnesota [4], showed that the bumps, knocks and sudden movements during sleep may signal onset of Parkinson's disease. The researchers found that nearly half of the people who performed in sleep the sudden movement, for 10 years had neurological disorders.

Researchers from the University of Toronto revealed a relationship between increased motor activity during sleep and the early disorders of the brain. In their opinion, if a person is dramatically spreads in a dream hands, moving her legs - in a word, performs a variety of involuntary movements of extremities, such behavior should be alerted and be the cause of going to the doctorthe neurologist, since the spread of pathology from the area of the brain that controls the physiology of sleep, for others its structure, it could trigger diseases such as Alzheimer's or Parkinson's disease [5].

We have made the assumption that the increased motor activity of the limbs of the patient at the time of his sleep can also be caused by epilepsy and thus, the analysis of this activity can be the basis, at least, for a more indepth studies in this direction.

\section{The need for instrument monitoring of epilepsy}

As can be seen from the presented material and by direct statements in the works now for a successful response required epilepsy monitoring system, or even a long instrumental monitoring which should be inexpensive in production and available during the operation, which can be used by the patient without medical personnel in the home that best suits obtaining ensemble of realizations under similar conditions. The results of research for their computer analysis can be provided by the patient, not only personally, but using IT, because the problem of lack of proper medical treatment for epilepsy is particularly relevant to residents of small settlements, especially in rural areas. So, according to $\mathrm{WHO}$, the risk of premature death in people with epilepsy in rural areas is three times higher than urban. In addition, the need for a prolonged instrumental monitoring is due not only control the tactics of therapeutic treatment, but also the need to minimize negative side-effects of drugs caused by the long course of their admission, especially in women of childbearing age.

\section{System analysis of paroxysmal accelerometry for monitoring of epilepsy in a sleep}

In accordance with the above, we have developed a system for the monitoring of epilepsy during sleep of a patient consisting of measuring device and motor activity recording and software for analysis of the information received.

The sensitive element of the device for measuring and recording motor activity is a triaxial accelerometer sensor (analog or digital) with a limit of measurement of $\pm 2 \mathrm{~g}$ (for example, ADXL327 or ADXL346 company Analog Divices) measurement signal which is processed by the microcontroller (e.g. Atmel ATmega328) and then recorded in a file on the microSD card. The device is powered from the element type is AAA, through step-up device to $3.3 \mathrm{~V}$. This choice of components and method of registration makes the device size slightly larger than, for example, a fitness tracker, but it is made to minimize the cost and operation of the device, and a method of registering data does not require the patient to any additional electronic gadgets and skills with him that makes our system accessible to all social classes of society. The patient is required only to fix the device on your wrist or ankle and turn it on before bed and off after sleep. Then the information from the card is transmitted to the specialist for computer analysis using application software, and the patient will be able to do this not only personally, but through any available means of telecommunication, such as Internet or mobile connection. This architecture of the system should significantly improve the quality of life of patients from small towns, as the lack of specialists in epileptology is particularly relevant for such locations, and in this case they will be able to obtain the necessary specialist advice without the need for personal visits to medical facilities, which may be at a considerable distance $[6,7]$.

The device received the Russia patent for useful model RU 168584 U1, 11.01.2016 [8].

Currently, with the help of our system comparative studies in 10 healthy individuals (control group) and 10 patients with an established diagnosis of "epilepsy".

For processing of the obtained results we took into account the main provisions for the analysis of nonstationary process with a single implementation, described in Chapter 12 [3]. For this one session night monitoring were divided into equal temporary files, which allowed us to obtain multiple implementations under the same conditions, and this allows for conventional statistical analysis, since short time intervals of the studied process can be considered quasi-stationary.

In the analysis of data about physical activity of patients in these groups during sleep, were identified following her graduation, numerically equal to the following: 
$n_{i}=\left\{\begin{array}{l}1,\left(A_{x}>0.15\right) \vee\left(A_{y}>0.15\right) \vee\left(A_{z}>0.15\right) \\ 0,\left(A_{x} \leq 0.15\right) \wedge\left(A_{y} \leq 0.15\right) \wedge\left(A_{z} \leq 0.15\right)\end{array}\right.$

$A$ - coefficient of motor activity; $T-$ one implementation; $t$-duration of interval partitioning; $U-$ the measured value of motor activity; $x, y, z$ - spatial coordinate axis; $\max$, $\min$ - maximum and minimum value; $A x, A y, A z$ - the relative peak amplitude peak-topeak.

If the value is $\mathrm{t}=60[\mathrm{~s}]$ and the condition (6), value (1) in healthy persons does not exceed $9 \%$, while $80 \%$ of individuals from this group it does not exceed $7 \%$, and persons from the first group it from $9 \%$ to $12 \%$ in individuals receiving therapeutic help and above $12 \%$ the rest. In our opinion, this provides a basis for a conditional division of motor activity when monitoring night's sleep on the following areas: to $7 \%$ - "green", from 7 to $9 \%$ "yellow", from 9 to $12 \%$ orange, from $12 \%$ - "red" and could be an indicator for the assessment of the patient by computer analysis of recorded data.

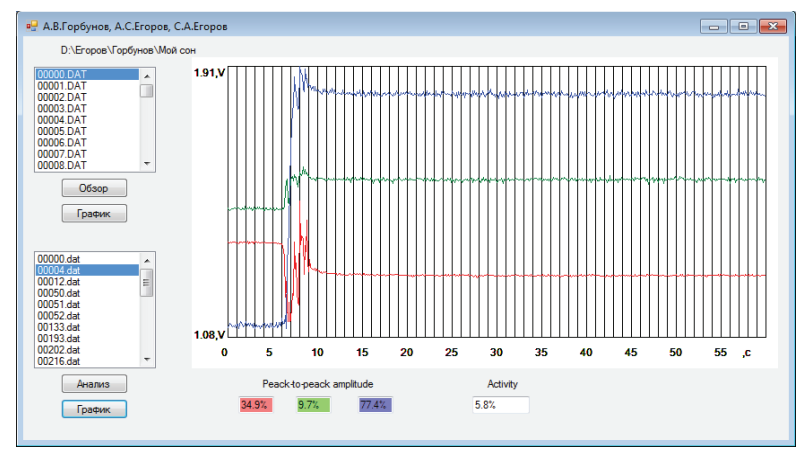

Fig.1. A patient in the "green" zone.

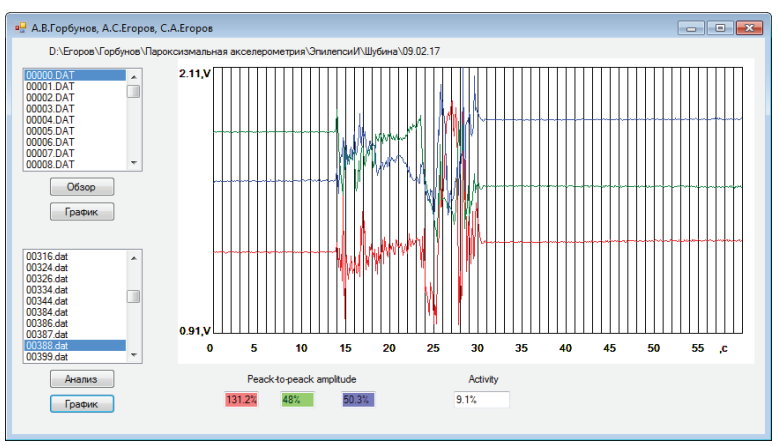

Fig.2. A patient in the "orange" zone.

It should be noted that the shape and duration obtained data are characteristic differences (Fig1, Fig.2).

\section{Summary}

We believe that our proposed system of analysis of paroxysmal accelerometry for monitoring epilepsy in the dream is promising for an effective anti-epileptic as as a complement to functional analysis, which will assist in the detection of the disease, and in the process of instrumental monitoring of treatment, especially in the regime of telemedicine. In our opinion, the division of motor activity during the monitoring of a night's sleep on the area to $7 \%, 7$ to $9 \%, 9$ to $12 \%$, more than $12 \%$ can be an indicator for the assessment of the patient by computer analysis of recorded data.

\section{References}

1. The website of the World Health Organization //URL:

http://www.who.int/mediacentre/factsheets/fs999/en $/ /(2017)$

2. The website of the Brown University, News from Brown, Asleep somewhere new, one brain hemisphere keeps watch// URL: https://news.brown.edu//2016/04/sleep ( 2016)

3. Julius S. Bendat, Allan G. Piersol, Random Data: Analysis and Measurement Procedures, (1986 by John Wiley \& Sons, Inc.)

4. The website of the University of Minnesota, Medical Bulletin. The pursuit of Parkinson's// https://www.med.umn.edu/news-events/medicalbulletin/pursuit-parkinson\%E2\%80\%99s (2017)

5. University of Toronto. "Potential cause of severe sleep disorder discovered, implications for Parkinson's disease." ScienceDaily. ScienceDaily, 15 June 2011. URL:http://www.sciencedaily.com/releases/2011/06 /110615103228.htm

6. S.V. Frolov, S.N. Makoveev, S.V. Semenova, S.G. Farea, Present-Day Tendencies in the Development of Medical Information Systems, Transactions of the TSTU V.16, (2010)

7. S.V. Frolov, M.A. Lyadov, O.A. Ostapenko, A.Yu. Potlov, I.A. Komarova, Automated Information 
System to Monitor Schoolchildren's Health, Development, Implementation Experience, Results, Transactions of the TSTU V.19, (2013)

8. A.V. Gorbunov, A.S. Egorov, S.A. Egorov, Patent RU 168584 U1, 11.01.2016 